\title{
Expression of Hoxa2 in cells entering chondrogenesis impairs overall cartilage development
}

Received September 5, 2005; accepted in revised form June 22, 2006

\begin{abstract}
Vertebrate Hox genes act as developmental architects by patterning embryonic structures like axial skeletal elements, limbs, brainstem territories, or neural crest derivatives. While active during the patterning steps of development, these genes turn out to be downregulated in specific differentiation programs like that leading to chondrogenesis. To investigate why chondrocyte differentiation is correlated to the silencing of a Hox gene, we generated transgenic mice allowing Cremediated conditional misexpression of Hoxa2 and induced this gene in Collagen 2 alpha 1-expressing cells committed to enter chondrogenesis. Persistent Hoxa2 expression in chondrogenic cells resulted in overall chondrodysplasia with delayed cartilage hypertrophy, mineralization, and ossification but without proliferation defects. The absence of skeletal patterning anomaly
\end{abstract}

Laurent Massip $^{1}$ · Jacques Picard · René Rezsöhazy ${ }^{2}(\nabla)$

Developmental Genetics Unit, Université catholique de

Louvain, Brussels, Belgium. Tel: +32 10473701

Fax: +32 10473717

E-mail: rezsohazy@vete.ucl.ac.be

Fabien Ectors

GIGA Liège, University of Liège, Liège, Belgium

Pierre Deprez · Mehdi Maleki · Catherine Behets · Benoît Lengelé

Experimental Morphology Unit, Université catholique de Louvain, Brussels, Belgium

Philippe Delahaut

C.E.R. Hormonology laboratory, Marloie, Belgium

\footnotetext{
${ }^{1}$ Present address: Centre de recherche en cancérologie CRHDQ, Université Laval, Québec, Canada.

${ }^{2}$ Present address: Unit of Veterinary Sciences, Department of Biology, Institut des Sciences de la Vie, Université catholique de Louvain, Louvain-la-Neuve, Belgium.
}

and the regular migration of precursor cells indicated that the condensation step of chondrogenesis was normal. In contrast, closer examination at the differentiation step showed severely impaired chondrocyte differentiation. In addition, this inhibition affected structures independently of their embryonic origin. In conclusion, for the first time here, by a cell-type specific misexpression, we precisely uncoupled the patterning function of Hoxa2 from its involvement in regulating differentiation programs per se and demonstrate that Hoxa2 displays an anti-chondrogenic activity that is distinct from its patterning function.

Key words Hoxa2 - Hox · chondrogenesis . chondrocyte - patterning - transgenesis . chondrodysplasia

\section{Introduction}

The vertebrate skeleton is a composite organ constituted of limited membranous structures arising by direct differentiation of mesenchyme into bone and a majority of endochondral elements formed from a preliminary cartilaginous anlage. According to the precursor tissue, one can distinguish the appendicular skeleton, which derives from lateral mesoderm, the axial skeleton, originating from paraxial mesoderm, and the cephalic skeleton, essentially derived from the neural crest (NC) cells (Couly et al., 1993; Lengelé et al., 1996; Christ et al., 2000; Santagati and Rijli, 2003). These precursors are of distinct embryonic origin as paraxial and lateral mesoderm both arise from a primitive mesodermal layer, whereas NC cells spread out from neural ectoderm to give the so-called ectomesenchyme.

After mesenchymal transformation of the sclerotome, budding of limb mesenchyme, or sprouting of 
skeletogenic NC cells, chondrogenic condensation occurs. This process is initiated by the migration and aggregation of precursor cells under the influence of fibroblast growth factors (FGFs) and bone morphogenic proteins (BMPs). The precise shape and location of condensations are mostly defined by the expression pattern of homeotic (Hox) genes (Christ et al., 2000). Condensation also requires $\operatorname{Paxl}$ and $\operatorname{Pax} 9$ gene products that allow precursor cells to respond to inductive signals such as Sonic Hedgehog (Shh) and prevent them from either apoptosis or premature differentiation (Peters et al., 1999). Recently, Pax 1 and -9 have been shown to be required for the activation of Nkx-3.2 (Bapxl), a Shh-induced factor, which in turn activates the chondrogenic marker Sox9 (Zeng et al., 2002; Rodrigo et al., 2003). The critical activation of Sox9 then marks the onset of chondrogenic differentiation. Indeed, if Sox9 is inactivated after the condensation step, cartilage fails to develop (Akiyama et al., 2002).

Once at the right place, condensations grow and cells differentiate to become proliferating chondrocytes. While the proliferation of precursor cells is notably controlled by factors of the retinoblastoma $(\mathrm{Rb})$ family, namely p107 and p130 (Rossi et al., 2002), chondrocytic differentiation still depends upon FGF (Murakami et al., 2000), BMP (Wan et al., 2001), as well as on ephrins (Stadler et al., 2001) and many other factors (Hoffmann et al., 2002; Scheijen et al., 2003). Further to their proliferation, chondrocytes become hypertrophic, cartilage undergoes mineralization, is resorbed, and replaced by bone. Here again, Sox9, but also the Wnt, Indian Hedgehog, and parathyroid hormone signaling cascades, coordinate these maturation steps (Yang et al., 2003).

Hox homeotic genes are well-known patterning genes that confer identity to skeletogenic condensations (Krumlauf, 1994). These genes are globally expressed in condensing cells but are turned off as differentiation starts. Hoxa2, the most anteriorly expressed Hox gene, contributes to the specification of the second branchial arch (BA2). Indeed, Hoxa2 inactivation results in the homeotic transformation of BA2, which therefore gives rise to the mirror duplication of BA1 structures (Gendron-Maguire et al., 1993; Rijli et al., 1993). Conversely, ectopic expression of Hoxa2 in the first BA of fish, frog, and chick leads to the reverse transformation (Grammatopoulos et al., 2000; Pasqualetti et al., 2000; Hunter and Prince, 2002). The homeotic transformation of BA2 in Hoxa2 knock-out (KO) mice is paralleled by the activation of the BA1specific markers Ptxl and Lhx6. In addition, the phenotype of the Hoxa2 $\mathrm{KO}$ is partially rescued in Hoxa2/Ptxl double KO. It was thus proposed that Hoxa2 confers the BA2 fate by restricting Ptx1 and Lhx6 expression domains anteriorly to BA2 in wild-type (WT) embryos. As Ptxl and Lhx6 activation in BA1 is under the control of FGFs, Hoxa2 activity was suggested to interfere at some point with the FGF signaling cascade (Bobola et al., 2003).

While previous studies on Hoxa2 function basically addressed its roles in the patterning of embryonic structures, the link between Hoxa2 activity and differentiation programs per se remains elusive. In particular, the reason why, once properly located and specified, cartilage precursors turn their Hox genes off remains unclear. Interestingly, Sox 9 and $C b f a l$ expression domains have been reported to be extended in Hoxa2 KO mice (Kanzler et al., 1998). As these genes are well-known chondro- and osteogenic lineage markers, this phenomenon was first interpreted as the loss of a putative Hoxa2 anti-skeletogenic activity in the BA2 area. However, in both the Hoxa2 KO and Hoxa2 ectopic expression experiments (Grammatopoulos et al., 2000; Pasqualetti et al., 2000), it was not possible to discriminate whether the phenotypic alterations were imputable to the involvement of Hoxa2 in the patterning of developing structures or to its possible effect on differentiation programs of cartilage and bone per se.

To investigate specifically why Hoxa2 needs to be switched off by the onset of chondrocyte differentiation, we analyzed the impact of a sustained Hoxa2 activity on cartilage development. In that respect, we generated transgenic mouse lines allowing Cre-mediated Hoxa2 induction and we crossed these lines with mice bearing a Cre recombinase gene under the control of the cartilagespecific Col2al promoter (Ovchinnikov et al., 2000). Thereby, we caused Hoxa 2 expression to persist beyond the onset of chondrocyte differentiation, i.e., beyond that precise stage it should be turned off, and to extend to larger territories than its normal sphere of action. Induced mice display delayed cartilage development and severe chondrodysplasia. Histological characterization of developing cartilaginous elements in these mice further demonstrates that Hoxa2 activity impairs chondrogenesis from its earliest steps. As this downregulatory effect is exerted after the condensation step, we propose that it is independent of the patterning function of Hoxa2 but rather results from the interference with a common basic chondrogenic program.

\section{Methods}

Plasmid constructs

The transgene for the conditional expression of Hoxa2 was obtained as follows: a Hoxa 2 genomic fragment spanning $47 \mathrm{bp}$ of the $5^{\prime}$ untranslated region (UTR) plus the entire coding sequence was inserted downstream of a $4.3 \mathrm{kbp} E c o R I-A l u I$ fragment of the human $\beta$-actin promoter including the first intron (IVS1) of the gene (Leavitt et al., 1984; Fig. 1A). This fragment was cloned upstream of a $4.4 \mathrm{kbp}$ reporter cassette. The reporter cassette contains a $1.1 \mathrm{kbp}$ internal ribosome entry site (IRES) from Theiler's virus (Shaw-Jackson and Michiels, 1999) fused to a nuclear localizing sequence (NLS)-tagged Escherichia coli lac $Z$ gene and followed by the SV40 late region polyadenylation signal. A 
loxP-flanked cassette to arrest transcription (STOP) was inserted between the $\beta$-actin promoter and Hoxa2 sequence. The stopping cassette was obtained from pBS302 (Invitrogen, Carlsbad, CA) after addition of a neomycin resistance gene (Kellendonk et al., 1996) to a unique BglII site. Introduction of the stopping cassette into the transgene was performed in vitro, using the Crerecombinase system (Clontech, Mountain View, CA; construct details available upon request).

\section{Generating and maintenance of transgenic mice}

SpeI and/or SfiI linearized transgenes were microinjected into the male pronucleus of inbred FVB zygotes and transferred into $\mathrm{C} 57 \mathrm{Bl} /$ $6 \times \mathrm{C} 3 \mathrm{~h} \mathrm{F1}$ foster mice at E0.5. Transgenics were identified by polymerase chain reaction (PCR) screening using specific primers for "fragment 2" (Figs. 1A,1B). Transgenics were mated with mice for a collagen II $\alpha 1$ promoter-directed Cre-expression (Col2al-Cre; Ovchinnikov et al., 2000). Col2a1-Cre and Col2a1/Hoxa2-lacZ simple and double transgenics were mated with $R 26 R$ mice (Soriano, 1999) for Cre-activity detection. Excision of the STOP cassette was assayed with PCR primers for "fragment 1" (Figs. 1A,1B).

\section{Western blotting}

To evaluate the level of Hoxa2 expression upon Cre-mediated induction, E13.5 embryos were collected and individually processed for whole protein extraction. Thirty micrograms of each protein extract, as estimated by the Pierce bicinchoninic acid protein assay kit (Pierce Biotechnology Inc., Rockford, IL), were run on sodium dodecyl sulfate-polyacryilamide gel electrophoresis and transferred to a nitrocellulose membrane. Immuno-detection of Hoxa2 was performed with the goat polyclonal anti-Hoxa2 antibody (N-20, sc17149; Santa Cruz Biotechnology Inc., Santa Cruz, CA) at a 1:20,000 dilution, followed by a secondary reaction with the peroxidase-coupled anti-goat immunoglobulin (IgG; PI-9500; Vector Laboratories, Burlingame, CA) used at 1:200. Detection of $\beta$-actin involved the rabbit anti-actin (20-33) antibody (A5060; Sigma, Bornem, Belgium) at 1:8,000 and the biotinylated antirabbit IgG (BA-1000; Vector) at 1:200 as a secondary antibody. Western blots were revealed by chemo-luminescent reactions (Pierce Supersignal West Pico trial kit) and scanned for quantification of detected proteins (EPSON Perfection 4990 Photo; data treatment with Image $\mathrm{J}$ v1.345 software). A semi-quantitative estimate of the level of Hoxa2 expression was obtained by comparison with the level of $\beta$-actin expression.

\section{Whole-mount staining}

For bone and cartilage staining, after removal of the skin and internal organs, embryos were fixed overnight in $95 \%$ ethanol, stained with $0.02 \%$ alcian blue in $4: 195 \%$ ethanol:acetic acid for 2 days, washed in $95 \%$ ethanol, immersed in $2 \% \mathrm{KOH}$ for $18 \mathrm{hr}$, stained in $75 \mathrm{mg} / \mathrm{ml}$ alizarin red in $1 \% \mathrm{KOH}$ overnight, processed through a graded series of glycerol in ethanol and stored in $100 \%$ glycerol.

For cartilage staining, E13.5 embryos were fixed overnight in Bouin's solution, rinsed briefly with water, immersed for at least $1 \mathrm{hr}$ in four changes of $1 \%$ ammonia in $70 \%$ ethanol, and stained with $0.05 \%$ alcian blue in $5 \%$ acetic acid. Embryos were rinsed three times in $5 \%$ acetic acid for $1-2 \mathrm{hr}$ each and then once overnight. Specimens were dehydrated through graded ethanols, cleared, and stored in 2:1 benzyl alcohol:benzyl benzoate (BA:BB; Sigma).

For $\beta$-galactosidase activity detection (X-Gal staining), embryos harvested from E9.5 to 15.5 were fixed up to $60 \mathrm{~min}$ in $4 \%$ paraformaldehyde (PFA) and washed in $0.01 \%$ sodium deoxycholate, $0.02 \%$ NP-40 in phosphate-buffered saline (PBS; $\mathrm{pH} 7.4$ ). Specimens were then stained overnight at room temperature in washing buffer containing $1 \mathrm{mg} / \mathrm{ml} \mathrm{X-gal} \mathrm{(Sigma),} 5 \mathrm{mM}$ potassium ferrocyanide, $5 \mathrm{mM}$ potassium ferricyanide, and $2 \mathrm{mM} \mathrm{MgCl}_{2}$. Samples were subsequently rinsed, post-fixed in 4\% PFA for $15 \mathrm{hr}$, dehydrated, and eventually processed through histology or cleared in $\mathrm{BA}: \mathrm{BB}$.

Histology and immunohistochemistry

Most embryos were fixed overnight in 4\% PFA in PBS before embedding in paraffin. They were cut into 5 - $\mu \mathrm{m}$-thick sections that were stained with hematoxylin, alcian blue-nuclear fast red, or according to Von Kossa's procedure.

Immunohistochemical detection of type-X collagen, proliferating cell nuclear antigen (PCNA), Hoxa2, Fgfr3, and Sox9 was performed with rabbit anti-collagen $\mathrm{X}$ (Calbiochem), anti-PCNA (Santa Cruz), anti-Hoxa2 (kindly provided by A. Nazarali), antiFgfr3 (Santa Cruz), and anti-Sox9 (Sigma) as primary antibodies, respectively. Immunoperoxidase staining was carried out using the Envision+ kit (Dako, Glostrup, Denmark). Endogenous peroxidase was blocked with $0.03 \%$ hydrogen peroxide for $5 \mathrm{~min}$. After three washes in distilled water, type-X collagen antigen was unmasked by 3 -min microwave treatment in citrate buffer $(10 \mathrm{mM}, \mathrm{pH} 6.0)$ and hyaluronidase from bovine testes $(2 \mathrm{mg} / \mathrm{ml}$ in PBS, pH 7.0, Sigma) at room temperature. Unmasking of PCNA was performed by $10 \mathrm{~min}$ microwave treatment. Thereafter, the nonspecific antigenic sites were neutralized by $10 \%$ normal goat serum (NGS) in PBS for $30 \mathrm{~min}$. The sections were then incubated overnight at $4{ }^{\circ} \mathrm{C}$ with the primary antibody (anti-collagen $\mathrm{X}, 1: 400$; anti-PCNA, 1:200; anti-Hoxa2, 1:800; anti-Fgfr3, 1:500; and antiSox $9,5 \mu \mathrm{g} / \mathrm{ml})$. Then, they were washed twice in PBS and incubated with secondary goat anti-rabbit antibody conjugated with a peroxidase-labeled polymer. After three changes in PBS, peroxidase was revealed by the diaminobenzidine reaction. The sections were counterstained with hemalun or alcian blue-nuclear fast red, dehydrated, and finally mounted. Control experiments included omission of the first antibody. PCNA-positive cells were counted in microscopic fields delimited by two successive intervertebral disks.

\section{Bone mineral density (BMD) determination}

Under anesthesia (Drobac et al., 2004), six Col2a1/Hoxa2-lacZ and six $\beta S$-Hoxa2-lac $Z$ mice were scanned with a peripheral quantitative computed tomography (pQCT) XCT Research SA+ (Stratec Medizintechnik GmbH, Pforzhein, Germany) 1 day after birth. The Col2a1/Hoxa2-lacZ mice were scanned a second time after death, the $\beta S$-Hoxa2-lac $Z$ mice after sacrifice at the equivalent time. Three $500-\mu \mathrm{m}$-thick midsagittal and paramedian sagittal slices spaced out of $250 \mu \mathrm{m}$ were selected from a scout view image. The total scan lasted about $15 \mathrm{~min}$. The voxel size was $70 \mu \mathrm{m}$. Images were analyzed with the XCT540 software provided with the pQCT. A $0.044 \mathrm{~mm}^{2}$ region of interest (ROI) was selected in the third cervical (C3), sixth thoracic (C6), and fourth lumbal (L4) vertebrae on the best slice of each mouse. BMD $\left(\mathrm{mg} \mathrm{HAP} / \mathrm{cm}^{3}\right)$ was measured for each ROI. The heights of three contiguous vertebrae of the cervical (C2, C3, C4), thoracic (T5, T6, T7), and lumbal (L3, L4, L5) regions were also measured with the software.

\section{Results}

Sustained Hoxa2 expression from the onset of chondrocyte differentiation results in a severe chondrodysplasia

To investigate why Hoxa2 needs to be silenced once chondrocyte differentiation goes on, a binary Cre-loxP 
recombination system was used to generate conditional Hoxa 2 expression in developing cartilage. We produced a construct that consists in the Hoxa2 genomic sequence placed under the control of the human $\beta$-actin promoter and translationally coupled to a lac $Z$ reporter by an IRES. This construct was further modified by inserting a "floxed" cassette blocking transcription between the $\beta$-actin promoter and the Hoxa2 coding sequence, to give rise to a silent $h \beta$-actin-STOP-Hoxa2-IRES-lac $Z$ transgene ( $\beta S$-Hoxa2-lac $Z$ for simplification, Fig. 1A). Cell transfection assays, followed by $\beta$-galactosidase activity detection demonstrated that the construct was correctly expressed in the absence of the blocking cassette. In addition, transgene expression upon cotransfection experiments resulted in specific transactivation of known target reporters, providing evidence that an active Hoxa2 protein was expressed from the construct (data not shown).

A
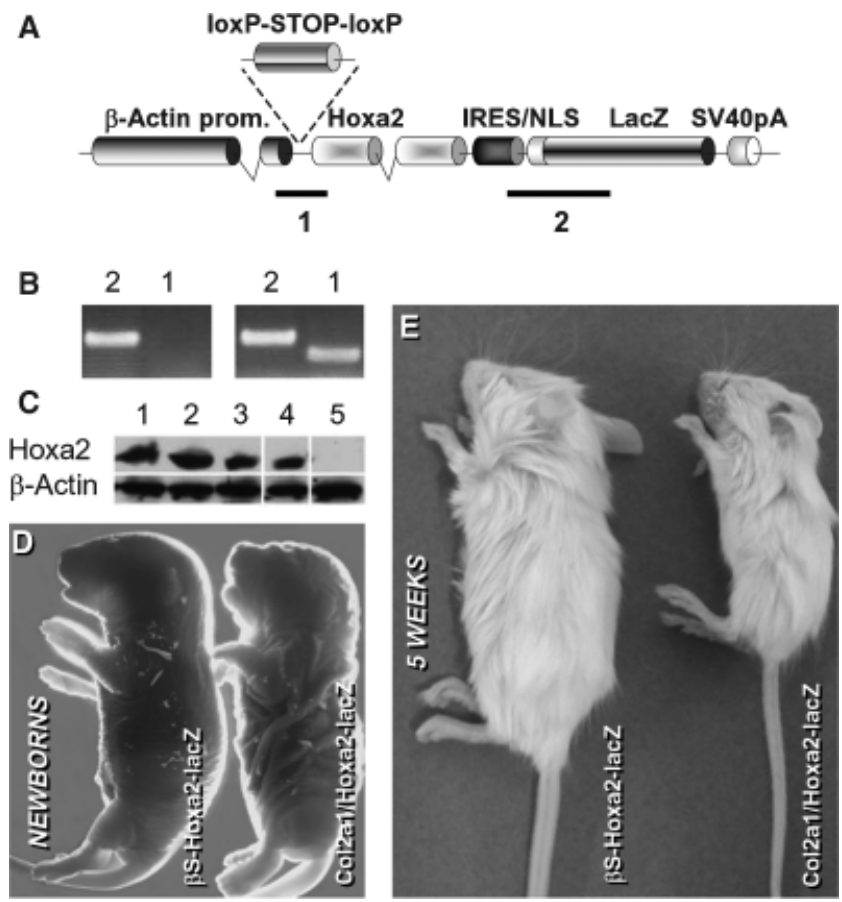

Fig. 1 Transgenic mice for the Col2al-Cre-induced expression of Hoxa2. (A) The transgene is composed of the Hoxa2 genomic sequence under the transcriptional control of the human $\beta$-actin promoter and followed by the internal ribosome entry site (IRES)nuclear localizing sequence (NLS)-lac $Z$ reporter sequence. A floxed cassette for transcriptional arrest (STOP) was inserted between the promoter and the Hoxa2 coding region. (B) Polymerase chain reaction (PCR) screening at the junctions between IRES and lac $Z$ yields fragment " 2 ," and allows detection of transgenic individuals. PCR with primers specific of the promoter and Hoxa2 sequences gives rise to fragment "1," when the STOP cassette has been removed by Cre-mediated recombination. (C) Western blotting of whole-protein extracts from E13.5 Col2al/Hoxa2-lacZ double transgenic embryos reveals Hoxa2 expression (lanes 1-4), while simple transgenics do not express Hoxa2 at a detectable level (lane 5). (D) At birth, the mostly affected transgenics for both Col2al-Cre and $\beta S$-Hoxa2-lac $Z$ transgenes die and show a crumpled skin with a shortened snout. (E) At 5 weeks, the mildly affected double transgenics show severe growth retardation.
The resulting $\beta S$-Hoxa2-lac $Z$ transgene was microinjected into mouse zygotes and transgenic progeny was identified by PCR. Among eight transgenic founders, two were retained and mated with a heterozygous Col2al-Cre strain that drives Cre-expression in differentiating chondrocytes (Ovchinnikov et al., 2000). As strikingly similar skeletal phenotypes were observed in the progenies of both founders, the derived lines will be indistinctly discussed hereafter. In these $\beta S$-Hoxa2-lac Z transgenic lines, Cre-mediated excision of the blocking cassette was confirmed by PCR (Fig. 1B). To ensure that the Hoxa2 protein was expressed upon inductive crossings, E13.5 embryos were collected and processed for whole-protein extraction and Western blotting. Extracts obtained from embryos bearing both the $\beta S$ Hoxa2-lacZ and Col2a1-Cre transgenes gave rise to a Hoxa2-specific signal (Fig. 1C). In contrast, Hoxa2 expression was not detected in extracts obtained from WT or simple transgenic embryos. A semi-quantitative estimate of the Hoxa2 protein revealed that the relative expression of Hoxa2 varied by less than a factor two among double transgenic individuals (data not shown).

Upon mating with Col2al-Cre mice, about one-fourth of the resulting double transgenics (Col2a1/Hoxa2-lacZ for simplification) died within the first few hours of life (17.5\% of double transgenics at birth versus $25 \%$ at E18.5; Table 1). They presented a crumpled skin with a kinked tail, a shortened snout, and frequent palatal clefts (Fig. 1D). Another quarter of the double transgenic littermates showed important growth retardation (Fig. 1E) and died within a few weeks. Finally, less than two-thirds of the double transgenics were viable, although mildly dwarf or malformed $(15.5 \%$ of double transgenics at 12 weeks post partum, Table 1).

$\mathrm{X}$-gal staining of E13.5 double transgenics confirmed specific transgene activation into a large set of developing cartilages (Figs. 2A,2B). E. Coli $\beta$-galactosidase activity was detected all along the axial skeleton, from the proximal Meckel's cartilage and otic capsule to the tail tip. Rib processes and limbs were also clearly stained with an intense signal in limb joints. The strongest signal was detected in the cervico-occipital and proximal Meckel's cartilage regions. Unexpectedly, Cre-recombinase also appeared to induce the $\beta S$ Hoxa2-lacZ transgene into the whole body dermis from E15 onwards (data not shown), which could account for skin abnormalities in double-mutant newborns.

Skeletons of E18 fetuses (nine double Col2a1/Hoxa2lac $Z$, five WT and five $\beta S$-Hoxa2-lac $Z$ transgenics) and neonates (11 double Col2a1/Hoxa2-lacZ, five WT and five $\beta S$-Hoxa2-lac $Z$ transgenics) were analyzed by differential staining of bones and cartilages. A global hypoplasia of both axial and appendicular elements was observed in all Col2a1/Hoxa2-lacZ mutants, while simple $\beta S$-Hoxa2-lac $Z$ transgenics were indistinguishable from WT littermates (Figs. 3A,3G). Col2al/ Hoxa2-lac $Z$ double transgenics displayed an overall 
Table 1 Proportion of Col2a1/Hoxa2-lacZ mice at different developmental and postnatal stages in Col2al-Cre $\times \beta S$-Hoxa2-lacZ progenies

\begin{tabular}{lll}
\hline Age & Animals (litters) & Col2al/Hoxa2-lacZ $(\%)$ \\
\hline E13.5 & $111(10)$ & $27(24.3)$ \\
E18.5 & $140(12)$ & $36(25.7)$ \\
Newborn & $114(10)$ & $20(17.5)$ \\
6 weeks & $76(7)$ & $12(15.8)$ \\
12 weeks & $71(7)$ & $11(15.5)$ \\
\hline
\end{tabular}

less intense cartilage staining and a reduced axial length. The vertebral column curvature was flattened with cervical lordosis (arrowhead) and Spina bifida (arrow). The number of vertebrae and ribs was normal (Figs. $3 \mathrm{~B}, 3 \mathrm{H})$, but ossification of the vertebral bodies appeared delayed, particularly in the cervical spine (arrowhead). The limbs were never malformed but slightly reduced in size and the ossification of the autopod was always delayed (Figs. 3C,3I). The skull presented either a reduced size or a brachycephaly (Figs. 3D,3J). With regard to paraxial mesoderm derivatives, the supraoccipital was rarely formed. The neural arches, vertebral bodies, basisphenoid, basioccipital, and exoccipital were always severely reduced (Table 2; Figs. 3D,3J). Interestingly, the malformed vertebral bodies were often fused to the neural arches (Figs. 3B,3H), and, in a similar way, the basioccipital was frequently joined to the exoccipitals (Figs. 3D,3J). Concerning the NC derivatives, the basihyoid, otic capsule (of dual origin), and middle-ear ossicles were reduced or poorly ossified but never malformed (Figs. 3E,3K). Meckel's cartilage was reduced, absent (Figs. $3 \mathrm{E}, 3 \mathrm{~K})$, or sometimes split in its proximal part. The nasal capsule, pterygoid, tympanic, and presphenoid bones were also clearly reduced. In some cases, the
A

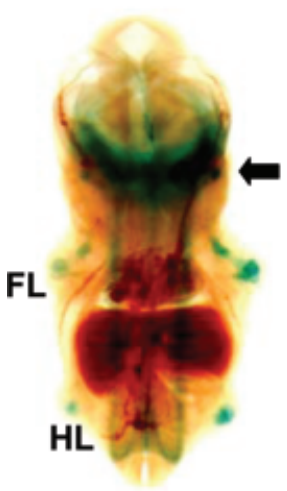

B

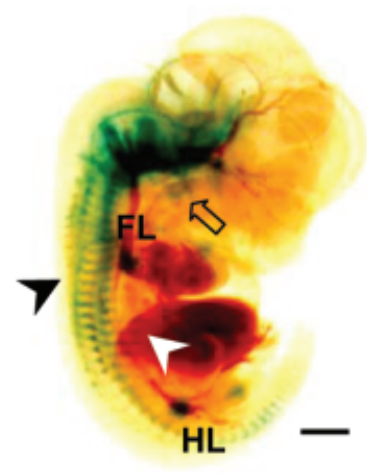

Fig. 2 X-gal staining of E13.5 Col2a1/Hoxa2-lacZ transgenics shows transgene expression in differentiating cartilages. $\beta$-galactosidase activity is detected (A) in forelimbs (FL), hindlimbs (HL), otic capsules (black arrow), as well as (B) in the whole vertebral column (black arrowhead), skull base, in the region of proximal Meckel (open arrow) and in differentiating ribs (white arrowhead; scale bar $=0.5 \mathrm{~mm})$. palatine bones were so dramatically reduced that the presphenoid was apparent (Table 2 and Figs. 3F,3L). The laryngeal and tracheal cartilages, the sternum, xiphoid process, and clavicles were normal, although slightly smaller. Most membranous bones of the face were also reduced so that actually all the examined skeletal structures appeared affected.

Col2al-expressing chondrogenic precursors are normally abundant and distributed

No homeosis was expected to be observed in Col2al/ Hoxa2-lacZ transgenics as only the differentiation step was targeted. Consistently, although clearly hypoplastic or locally interrupted, the proximal Meckel's cartilage and middle-ear ossicles appeared normally shaped in these pups. To further exclude any major patterning defect as a possible cause of the observed Col2al/ Hoxa2-lacZ mutant phenotype, we verified that chondrogenic precursor cells were normally distributed in the presence of both transgenes.

The $R 26 R$ reporter mouse is a strain that has been engineered to allow the early detection of Cre recombinase activity in tissues through lac $Z$ conditional expression (Soriano, 1999). Crossing the Col2al-Cre transgene in the R26R background therefore allowed the detection of the Col2al-expressing chondrogenic precursors. We further brought together the three transgenes ( $\beta S$-Hoxa2-lacZ, Col2al-Cre, and R26R) in the same pups and compared the distribution of precursor cells with that observed in controls (Col2alCre/R26R). Patterning defects or massive cell death arising upon Hoxa2 induction should display an altered abundance or distribution of Col2al-Cre-expressing cells in the presence of the $\beta S$-Hoxa2-lacZ transgene. Embryos were harvested at E10.5, when progenitor cells migrate from the somites and condense. At this stage, these cells express Col2al-Cre, as reported by Ovchinnikov et al. (2000). No obvious difference in stained cells' distribution was found between Col2a1/Hoxa2lac $Z(n=3)$ and Col2al-Cre $(n=3)$ transgenic embryos in the $R 26 R$ background (Figs. 4A,4B), indicating that cells were equally abundant and similarly patterned in the presence or absence of the Hoxa2-inducible transgene. To exclude the possibility that the similar precursor cell distribution in these embryos reflects a low penetrance of the skeletal phenotype in the $R 26 R$ genetic background, we looked at the skeletons of E18 fetuses in the progeny of identical crosses. Col2al/ Hoxa2-lac $Z$ double transgenics in the $R 26 R$ background $(n=7)$ showed exactly the same malformations at the same frequency as those reported in Table 2. These data therefore demonstrate that the phenotype resulting from the Col2al-Cre-mediated induction of Hoxa2 is not due to late patterning defects or massive cell death. 

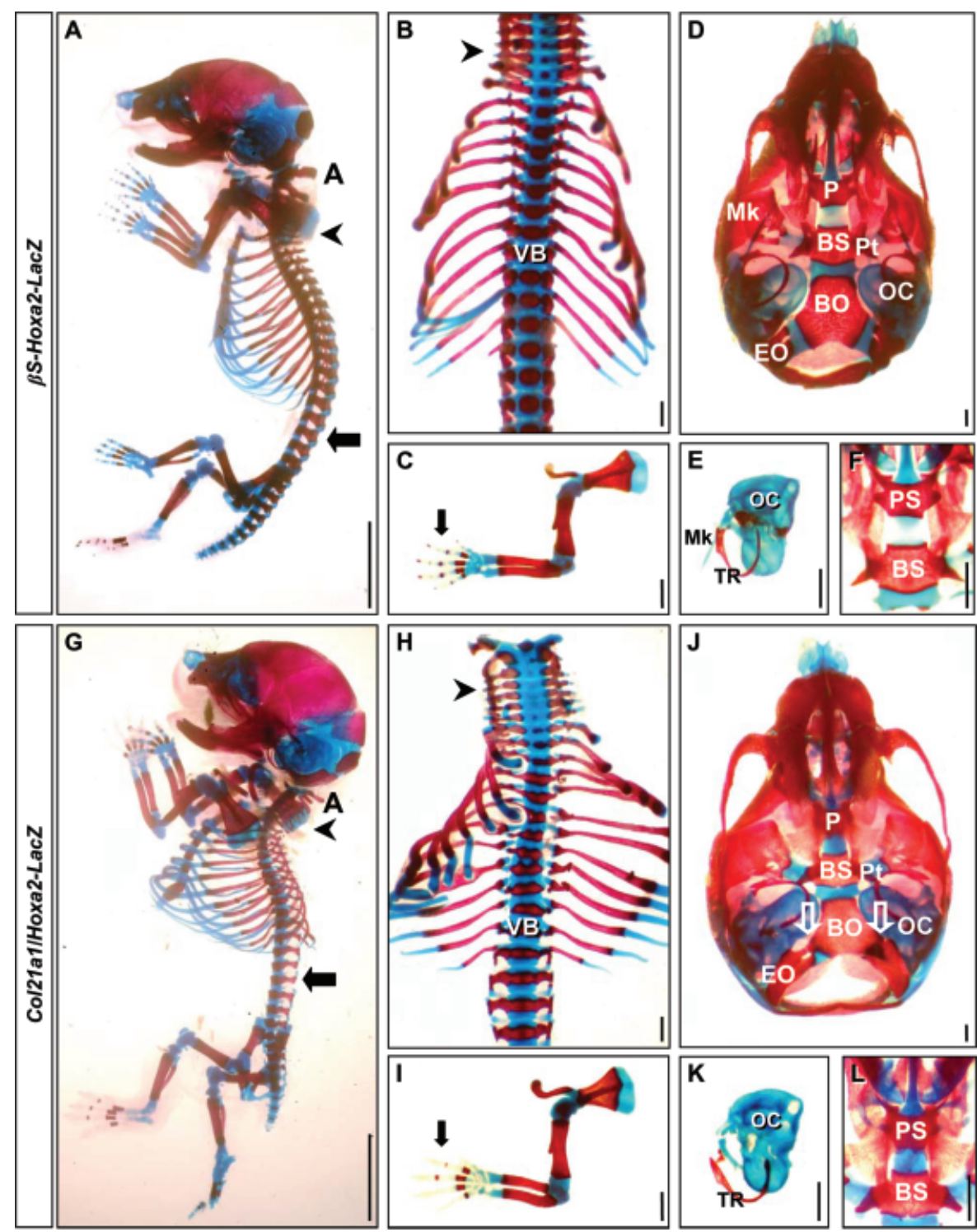

Fig. 3 Alcian blue and Alizarin red staining of Col2al/Hoxa2-LacZ and $\beta S$ Hoxa2-lacZ skeletons. (A,G) Differential staining of bones and cartilages reveals severe cervical lordosis (arrowhead) in newborn double mutants with spina bifida (arrow), and significant reduction of the cervical column, especially at the level of the atlas $(A$; scale bars $=5 \mathrm{~mm})$. (B,H) BS-Hoxa2-lacZ (B) and Col2a1/ Hoxa2-LacZ $(\mathrm{H})$ spines indicating global chondrodysplasia with aberrant shape of the vertebral bodies (VB) and reduced or absent ossification centers in Col2al/ Hoxa2-LacZ mutants (arrowheads; scale bars $=1 \mathrm{~mm}) . \quad(\mathbf{C}, \mathbf{I}) \quad \beta S$-Hoxa2-lacZ (C) and Col2a1/Hoxa2-LacZ (I) forelimbs showing size reduction in double-mutant limbs with delayed autopod ossification (arrow; $1 \mathrm{~mm}$ scale). (D,E,F,J,K,L) $\beta S$ Hoxa2-lacZ (D,E,F) and Col2al/Hoxa2LacZ $(\mathrm{J}, \mathrm{K}, \mathrm{L})$ skull bases and otic capsules (scale bars $=1 \mathrm{~mm}$ ). In the Col2al/ Hoxa2-LacZ mutants, the basioccipital (BO) is reduced and/or fused (arrows) to the exoccipitals (EO). The basi- (BS) and the presphenoid (PS) are reduced as well as the pterygoid $(\mathrm{Pt})$ and palatine bones (P), which are largely hypoplastic. The Meckel (Mk) is absent. Otic capsule (OC) shows an ossification delay, but is not malformed, like the tympanic ring (TR).
Overall cartilage maturation defects upon persistent Hoxa2 expression

To unravel the cause of the observed skeletal defects, each maturation step of skeletogenesis was addressed in Col2a1/Hoxa2-lacZ mutants. Histological and immuno-histochemical examination was carried out on Col2a1/Hoxa2-lacZ and $\beta$ S-Hoxa2-lacZ or WT E15.5 fetuses. At this developmental stage, proliferative, hypertrophic, mineralized, and ossified cartilages are visible in normal pups. In WT $(n=5)$ and $\beta S$-Hoxa2lac $Z$ fetuses $(n=4)$, bones and cartilage appeared well developed. In contrast, Col2a1/Hoxa2-lac $Z$ mutants $(n=7)$ showed chaotic cartilages organization with smaller differentiated hypertrophic areas (Figs. 4C,4D). Their vertebral column looked about half the normal size and spinous processes were hypoplastic or even absent. At E15.5, chondrocyte proliferation was examined through PCNA immunostaining. In both $\beta S$ Hoxa2-lacZ $(n=3)$ and Col2a1/Hoxa2-lacZ $(n=3)$ spines, proliferating cells were localized at the vertebral bodies periphery with a higher density around intervertebral disks (Figs. 4E,4F). No difference in proliferating cells number or localization was seen between simple- and double-mutant mice (Fig. 4G). The maturation of the hypertrophic cartilage was assessed by type-X collagen immunostaining. While an expected strong signal was precisely detected in the vertebral bodies centrum of WT $(n=2)$ and $\beta S$-Hoxa2-lacZ $(n=3)$ pups, it appeared milder and dispersed in double transgenics $(n=3$; Figs. 4H,4I). Finally, cartilage mineralization, emphasized by Von Kossa's/AR staining, was clearly delayed in double transgenics $(n=3)$ as compared with control pups $(n=3)$. It was even practically absent in the basihyoid (Figs. 4J,4K). All these observations were also noticed for double-mutant 
limbs and ribs, although they were less pronounced. In conclusion, while the proliferation of chondrocytes did not appear to be affected by a persistent Hoxa2 expression, the whole cartilaginous maturation process appeared impaired in Col2a1/Hoxa2-lacZ mice. In addition, Hoxa 2 misregulation equally affected skeletal derivatives of either NC or mesoderm origin.

Hoxa 2 expression in chondrogenic precursors impairs the initial differentiation steps

Whether the above-mentioned maturation defects could be related to an initial differentiation failure in Col2al/ Hoxa2-lac $Z$ mice remained to be addressed. To investigate on this issue, whole-mount double-mutant
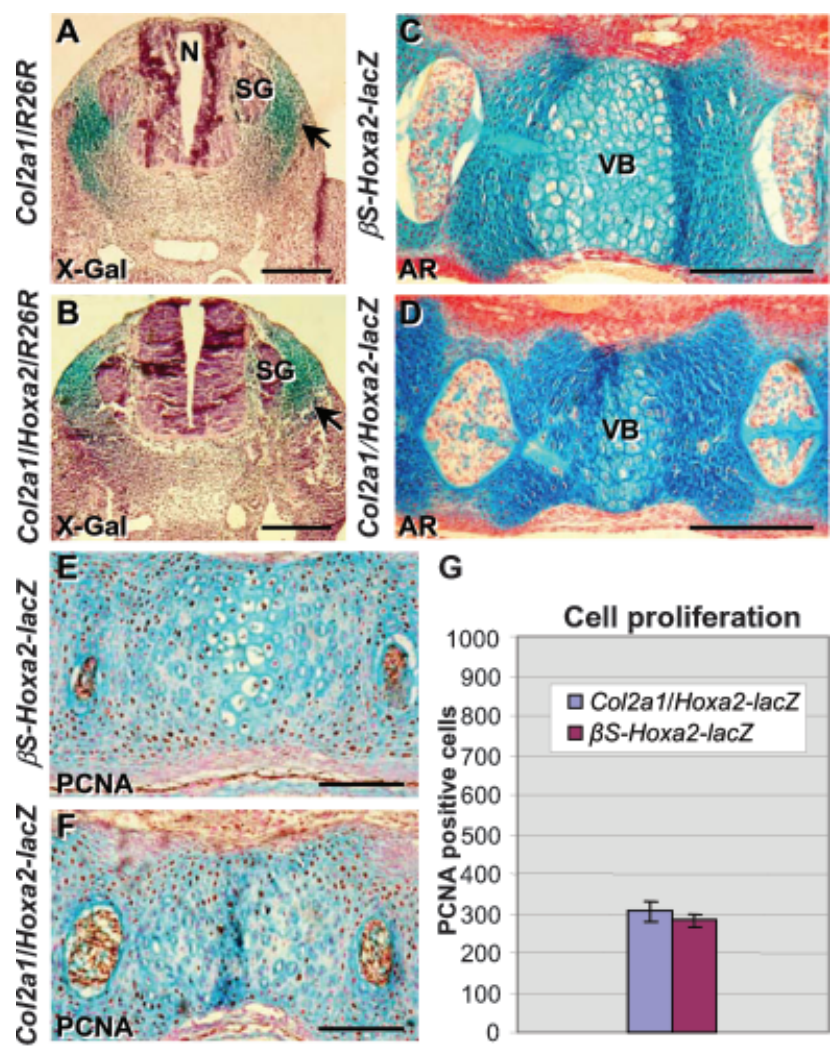

G
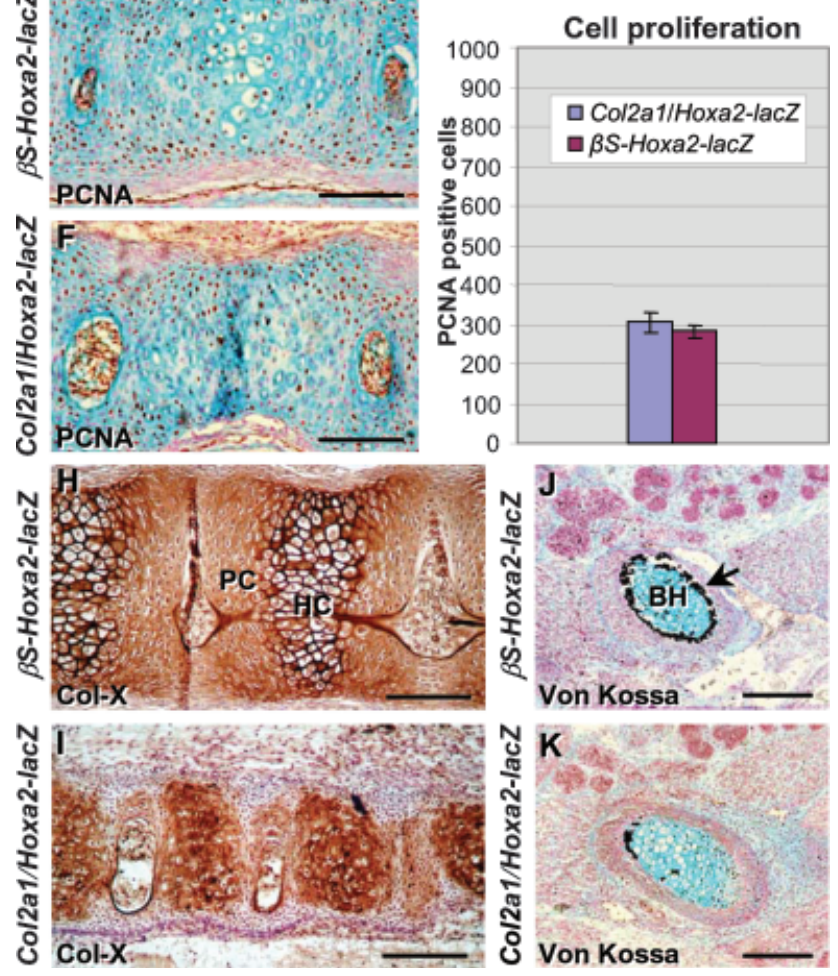

Table 2 Hypoplastic structures in Col2a1/Hoxa2-lacZ mutant neonates

\begin{tabular}{ll}
\hline Affected structures & Embryonic origin \\
\hline Nasal cartilage & Neural crest \\
Presphenoid & Neural crest \\
Basisphenoid & Paraxial (somitic) mesoderm \\
Basioccipital & Paraxial (somitic) mesoderm \\
Exoccipital & Paraxial (somitomeric) mesoderm \\
Supraoccipital & Paraxial (somitomeric) mesoderm \\
Otic capsule & Paraxial mesoderm + Neural crest \\
Incus and malleus & Neural crest (BA1) \\
Tympanic ring & Neural crest (BA1) \\
Pterygoid & Neural crest (BA1) \\
Palatine & Neural crest (BA1) \\
Meckel's cartilage & Neural crest (BA1) \\
Stapes & Neural crest (BA2) \\
Body of the hyoid & Neural crest (BA2) \\
Vertebrae & Paraxial (somitic) mesoderm \\
Ribs & Paraxial (somitic) mesoderm \\
Limbs & Lateral mesoderm
\end{tabular}

skeletons were examined at E13.5. At this stage, chondrocyte differentiation has progressed and hypertrophy has just begun in normal mice. Surviving Col2a1/Hoxa2-lacZ mice were thus mated together and embryos were collected. One resulting litter was genotyped and processed for alcian blue staining. Consistent with a mendelian distribution, 6/13 embryos were negative for at least one of the transgenes ( $\beta S$ Hoxa2-lacZ or Col2al-Cre). Those embryos presented a normal skeletal development, particularly in the verteb-

Fig. 4 Histological comparison between Col2a1/Hoxa2-lacZ double transgenics and Col2al-Cre littermates in the $R 26 R^{+/-}$ background at E10.5 (A,B), and histological and immunohistochemical comparison of Col2al/Hoxa2-lacZ mutants with $\beta S$ Hoxa2-lacZ littermates at E15.5 (C-K). In each case, $\beta S$-Hoxa2lac $Z$ transgenics showed the same phenotype as wild-type littermates. (A,B) X-Gal-stained transverse section of E10.5 embryos. Cre-expressing cell distribution (blue staining indicated by the arrow) in double Col2al-Cre/R26R transgenics (Col2al/ $R 26 R$ for simplification; A) and triple Col2al-Cre/ $\beta$ S-Hoxa2-lacZ/ $R 26 R$ transgenics (Col2al/Hoxa2/R26R for simplification; B), shows no difference between double mutants and control littermates. Neural tube (N), spinal ganglion (SG). (C,D) At E15.5, AR staining of a sagittal section in the lumbal column of $\beta S$-Hoxa2lacZ (C) and Col2a1/Hoxa2-lacZ (D) transgenics. Vertebral body (VB; E,F,G) proliferating cells staining (E,F) and counting $(\mathrm{G})$ via proliferating cell nuclear antigen (PCNA) immuno-detection in sagittal sections through cervical column of $\beta S$-Hoxa2-lac $Z$ (E) and Col2a1/Hoxa2-lacZ (F) transgenics. No significant difference was detected in proliferating cells number or localization between control and Col2a1/Hoxa2-LacZ mutant littermates. Each value shown in $(G)$ is the mean counted positive cells for three independent pairs of embryos. (H,I) At E15.5, anti-collagen X immunostaining on similar sections shows clear localization of collagen $\mathrm{X}$ in hypertrophic chondrocytes (HC) of vertebral bodies in $\beta S$-Hoxa2-lac $Z$ transgenics $(\mathrm{H})$ but fainter and less organized staining in Col2a1/Hoxa2-lacZ mutant (I). Proliferating chondrocytes (PC). (J,K) At E15.5, Von Kossa/AR staining of the basihyoid (BH) in $\beta S$-Hoxa2-lacZ (J) and Col2a1/Hoxa2-lacZ (K) transgenics showing a significant mineralization delay in double mutants compared with control pups (arrow). Scale bars $=200 \mu \mathrm{m}$. 

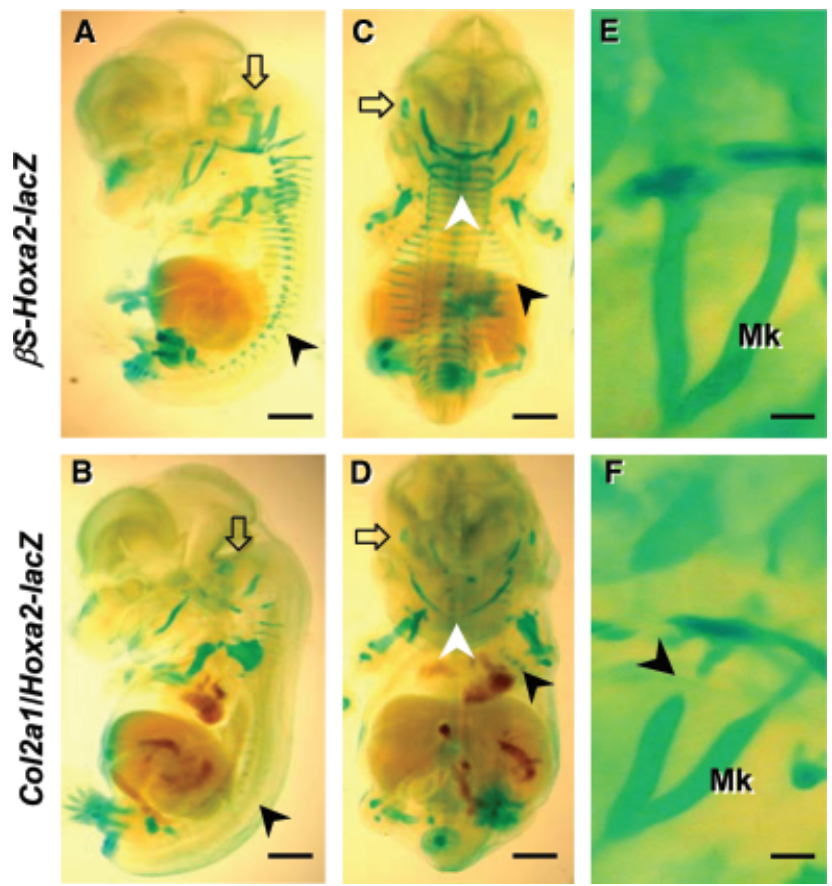

Fig. 5 Skeleton analysis of Col2a1/Hoxa2-lacZ and $\beta S$-Hoxa2-lacZ littermates at E13.5 by alcian blue staining of the whole cartilages. $(\mathbf{A}, \mathbf{B})$ Lateral view of $\beta S$-Hoxa2-lacZ (A) and Col2a1/Hoxa2-LacZ (B) transgenics showing exoccipitals (arrow) and cervical hypoplasia and no differentiation of the rest of the vertebral column (arrowhead). (C,D) Dorsal view of $\beta S$-Hoxa2-lacZ (C) and Col2al/ Hoxa2-LacZ (D) transgenics showing otic capsule (arrow) and Meckel (white arrowhead) hypoplasia and nearly no axial skeleton but distal ribs rudiments (black arrowhead). (E,F) Magnification of Meckel's (Mk) region in $\beta S$-Hoxa2-lacZ (E) and Col2a1/Hoxa2lac $Z(\mathrm{~F})$ transgenics. Split in the mutant Meckel's cartilage is shown (arrowhead in F). Scale bars $=0.5 \mathrm{~mm}(\mathrm{~A}-\mathrm{D})$ and $0.1 \mathrm{~mm}(\mathrm{E}, \mathrm{F})$.

ral column, ribs, basihyoid, otic capsule, and Meckel's cartilage (Figs. 5A,5C,5E). Conversely, the most affected double mutants (2/13) displayed almost no axial skeleton but rudiments of basioccipital, exoccipital, atlas, and distal ribs (Figs. 5B,5D). The Meckel's cartilage was occasionally split in its proximal part (Fig. $5 \mathrm{~F}$ ) and sometimes, the basihyoid was not yet differentiated. The remaining progeny $(5 / 13)$ showed an intermediate phenotype.

When looking at the X-gal staining intensity in E13.5 litters, the embryos showing the highest staining also displayed the most affected structures (data not shown). This correlation is suggestive of a gene dosage effect on the expressivity of the phenotype, which would explain the occurrence of a low number of more severely affected embryos interpreted as homozygous for the induced $\beta S$-Hoxa2-lac $Z$ transgene. This could also explain the apparent co-localization of Meckel's cartilage splitting point with an intense X-gal staining spot (compare Figs. 2B,5F). In contrast, staining was never detected in some rostral-affected structures like the presphenoid or the nasal capsule, which might display a higher sensitivity to Hoxa2 misexpression.
To further delineate the impact of the sustained Hoxa2 expression on the chondrogenic cascade, we looked at the expression of two of the earliest chondrogenic determinants, FGF receptor 3 (Fgfr3) and Sox9. Fgfr3 and Sox9 are both expressed at the very onset of differentiation and their expression persists up to the prehypertrophic stage. As Fgfr3 indirectly stimulates the expression of Sox9 (Murakami et al., 2000), an alteration in their relative expression domains would mean that Hoxa2 interferes at some point within this cascade in Col2a1/Hoxa2-lacZ mice. As expected, Hoxa2 was only detected in the vertebral column of Col2a1/Hoxa2-lacZ mutants. This staining was prominently found in undifferentiated cells lying at the periphery of restricted developing cartilages (Figs. 6A-6C). In both WT $(n=2)$ and $\beta S$-Hoxa2-lac Z $(n=2)$ E13.5 embryos, strong immunostaining signals for Sox 9 and Fgfr3 were observed in differentiating vertebral bodies (Figs. 6D,6F). Strikingly, upon Hoxa2 induc-
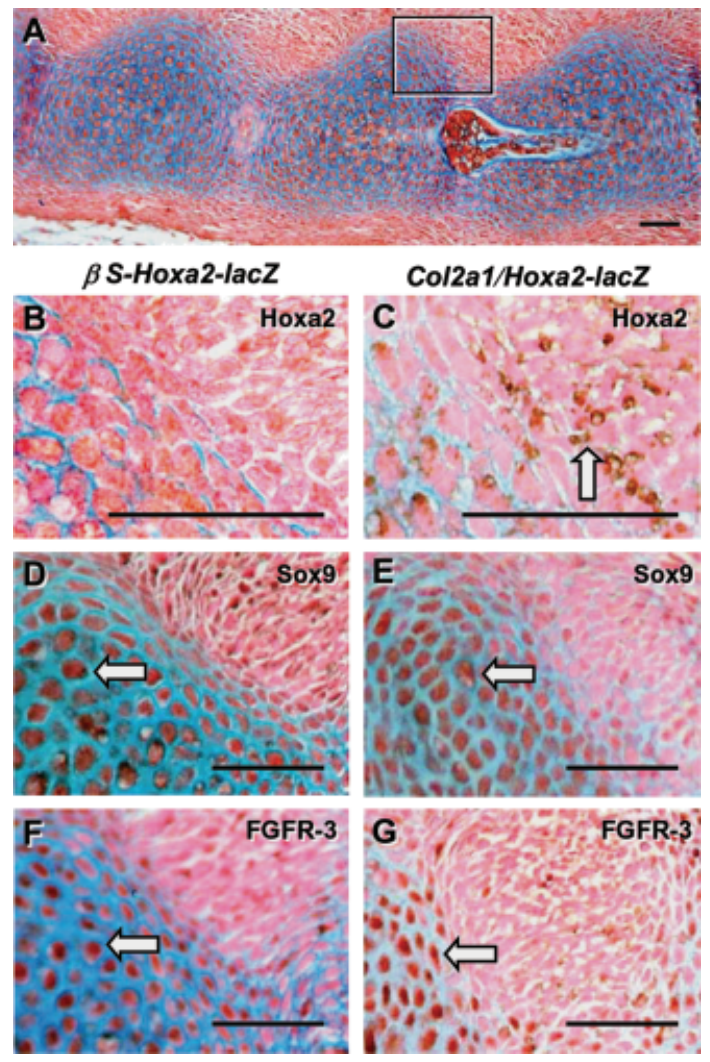

Fig. 6 Immuno-histochemical detection of Hoxa2, Sox9, and Fgfr3 expression on sagittal sections of the lumbar column of Col2al/ Hoxa2-lac $Z$ double transgenics and $\beta S$-Hoxa2-lac $Z$ littermates at E13.5. (A) Negative immunostaining in a $\beta S$-Hoxa2-lac $Z$ embryo. The framed area corresponds to the regions illustrated in $\mathbf{B}-\mathbf{G}$. In each case, $\beta S$-Hoxa2-lac $Z$ transgenics $(\mathrm{B}, \mathrm{D}, \mathrm{F})$ showed the same phenotype as wild-type littermates. (B,C) Hoxa2 is only detected in Col2a1/Hoxa2-lac Z transgenics (C) where it localizes mainly at the periphery of the differentiating condensations (arrow). (D,E) Sox9 and $(F, G)$ Fgfr3 are detected in differentiated chondrocytes (arrows) in both Col2a1/Hoxa2-lacZ and $\beta S$-Hoxa2-lacZ littermates. Scale bars $=50 \mu \mathrm{m}$. 
tion, detection of both Fgfr3 and Sox9 was equally limited to those restricted cartilaginous areas $(n=2$; Figs. 6E,6G), thereby suggesting that the downregulatory effect of Hoxa2 on cartilage differentiation takes place upstream of both these chondrogenic players.

Altogether, our results indicate that persistent Hoxa 2 expression in precartilaginous primordia impairs the very initial chondrogenic differentiation process and that chondrogenic precursors, whatever their NC, paraxial, or lateral mesoderm origin, are equally affected. This global impairment of differentiation would not result from a direct inhibition of $\operatorname{Sox} 9$ expression but rather grounds upstream of the stimulatory FGF signaling occurring at the onset of the chondrogenic program.

The Hoxa2-induced impairment of chondrogenesis does not affect further ossification

As already mentioned, the surviving double transgenics appear smaller than the unaffected simple transgenic or WT littermates. This difference was progressively marked after birth and appeared the most apparent by postnatal day 5 (vertex-sacrum size: double mutants to control ratio of $0.78 \pm 0.07 ; n=4)$. The overall size difference between double transgenics and control animals could be correlated to size differences in equivalent vertebrae. The height of nine vertebrae (C2-C4, T5-T7, and L3-L5) of double transgenics was measured 1 day after birth and on the day of their death (postnatal days 5, 28, 51, and 56) and compared with those of control littermates at the same age. The relative vertebral size was decreased in Hoxa2 expressers as compared with controls (axial size ratio of $0.63 \pm 0.26 ; n=12$ ). This postnatal growth defect caused by the expression of Hoxa2 in chondrocytes is consistent with the differentiation defect observed at fetal stages.

To address whether the impaired chondrogenesis was followed by a deficit in ossification per se or not, BMD was determined by pQCT in the C3, T6, and L4 vertebrae of those individuals for which the vertebral sizes were compared. BMD of Col2a1/Hoxa2-lacZ mutants did not appear significantly different from control littermates $\left(383.1 \pm 154.0 \mathrm{mg} / \mathrm{cm}^{3}\right.$ in double mutants versus $312.9 \pm 104.6 \mathrm{mg} / \mathrm{cm}^{3}$ in controls, $n=12$ ), providing evidence that the Hoxa2-mediated chondrodysplasia did not affect the subsequent ossification process as such.

\section{Discussion}

In the mouse, cartilages start to develop from mesenchymal condensations, while the expression of patterning
Hox genes expression turns off. This temporal sequence of events underlies an unresolved functional link between the fading down of Hox expression and the onset of chondrocyte differentiation. In this study, we generated transgenic mice that allow conditional Cremediated ectopic and heterochronic expression of Hoxa2. These mouse lines were used to investigate why such a Hox gene needs to be down-regulated to engage chondrogenic differentiation programs. To this end, we induced Hoxa2 activity beyond the onset of chondrocyte differentiation and analyzed the impact of a sustained expression of Hoxa2 on cartilage development. We therefore demonstrated that Hoxa 2 activity has a drastic down-regulatory effect on chondrocyte differentiation, which results in chondrodysplasia of $\mathrm{NC}$, sclerotome, and lateral mesoderm-derived skeletal elements.

Col2a1/Hoxa2-lacZ double transgenic mice show reduced or malformed secondary palate, nasal capsule, neurocranium, viscerocranium, otic capsule, vertebral column, ribs, and limbs. The lac $Z$ staining of precursor cells expressing Col2al revealed that their distribution was unaffected in the double mutants. Immunostaining of proliferating cells at E15.5 further showed that chondrocytic proliferation was also unaffected. However, alcian blue staining of early developing cartilage supported that the chondrodysplasic phenotype can be interpreted as resulting from an initial differentiation failure. At E13.5, while WT embryos display a wide cartilaginous skeleton, the most affected double mutants lack axial skeleton structures but only show occipital, cervical, distal ribs, or otic capsule rudiments. As a possible consequence, at E15.5, the skeleton of the affected individuals presents a severe maturation delay during the hypertrophy, mineralization, and ossification steps. Finally, ectopic Hoxa2 expression during chondrogenesis led to perinatal or early lethality for more than one-third of the double transgenic individuals. This variation in survival does not seem to reflect differences in the level of the Hoxa2 expression upon Cre-mediated recombination. Indeed, the semi-quantitative estimate of Hoxa2 expression in double transgenic E13.5 embryos did not reveal gross variation in Hoxa2 expression level. Rather, the susceptibility to early lethality may be due to the relative genetic heterogeneity of the offsprings of crossings between Colzal-Cre and $\beta S$-Hoxa2-lacZ lines. In fact, these transgenes have been maintained in distinct, C57BL/ 6xSJL hybrid (Ovchinnikov et al., 2000) and FVB inbred genetic backgrounds, respectively.

Previously reported ectopic and sustained expression of Hoxa2 led to distinct phenotypes according to the cell populations that were targeted for Hoxa2 misexpression and to the experimental design leading to different timing and persistence of Hoxa2 expression. Some ectopic activation of Hoxa2 resulted in patterning modifications but did not give rise to differentiation 
defects as such (Grammatopoulos et al., 2000; Pasqualetti et al., 2000). Other misexpression experiments did not lead to homeotic transformations (Grammatopoulos et al., 2000; Creuzet et al., 2002), but rather to a deficit in skeletal development. As previously discussed (Grammatopoulos et al., 2000; Pasqualetti et al., 2000; Creuzet et al., 2002), the different outcomes of Hoxa2 misexpression might reflect that in some instances, both $\mathrm{NC}$ cells and branchial arch host tissue were targeted while in others, Hoxa2 was only misexpressed in the NC. However, critical differences between these experiments also reside in that Hoxa2 misexpression was either transient or sustained.

Although our data parallel those obtained when only the NC cells were targeted to express Hoxa2 ectopically (Grammatopoulos et al., 2000; Creuzet et al., 2002), these experiments should be compared cautiously. In the work presented here, the timing of Hoxa2 misexpression and the cellular population that ectopically expressed this gene were clearly different from the previously reported experiments. In fact, by inducing Hoxa2 expression in Col2al-expressing cells, NC and branchial arches are allowed to be normally patterned. Hoxa 2 was thus precisely misexpressed only in cells that are normally committed to enter chondrogenesis and only at the precise stage chondrocyte differentiation goes on. This implies that not only the skeletogenic NC but also other skeletogenic compartments, i.e., paraxial and lateral plate mesoderm, were targeted and consistently showed chondrodysplasia. Altogether, our results therefore show that on a cell-autonomous basis, Hoxa2 persistent expression delays chondrocyte differentiation, by acting just upstream of a common basic chondrogenic program. As the selective down-regulation of the chondrogenic cascade occurs after migration of the precursors, Hoxa 2 is proposed to fulfill distinct but yet related roles during skeletal development. It could not only prevent the expression of some BA1-specific patterning genes, like Lhx6 or Ptxl, in BA2 structures (Bobola et al., 2003) but also impede premature chondrogenesis of these structures.

While patterning defects were observed in the Hoxa2 loss of function, no precocious skeletal differentiation or maturation was reported. This suggests that, in the absence of Hoxa2, early differentiation or maturation of incorrectly patterned structures or normal skeletal elements is kept under control of redundant modulators of chondrogenesis. In this regard, inactivation of the Pbxl gene coding for a key cofactor of Hox proteins resulted in premature cartilage maturation and ossification (Selleri et al., 2001). This indicates that the activity of other down-regulators of chondrocyte differentiation, like that of Hoxa2, relies on a partnership with Pbx1. Finally, AP-2 factors are required to provide Hoxa2 expression in the skeletogenic $\mathrm{NC}$ (Maconochie et al., 1999). Interestingly, AP-2 $\alpha$ was recently shown to inhibit chondrogenesis in ATDC5 cells (Huang et al., 2004), which probably involves Hoxa2 as a target to mediate this anti-skeletogenic effect in vivo.

Extension of the Sox9 expression domains into Hoxa2 $2^{-/-}$skeletogenic territories has been reported and the forced expression of Sox9 under the control of the Hoxa2 promoter partially phenocopied the Hoxa2 KO (Kanzler et al., 1998). Sox9 was thus proposed to be negatively regulated by Hoxa2. Consistently, similar chondrodysplasia with split Meckel's cartilage was found in Sox9 haploinsufficient mice (Bi et al., 2001) and complete inactivation of a floxed Sox9 allele in the limbs from the onset of differentiation blocked the chondrogenic cascade (Akiyama et al., 2002). Here, we show that in Col2al/Hoxa2-lacZ mutant skeletons, Sox9 and Fgfr3 expression domains are reduced. As Fgfr3 has been shown to stimulate Sox9 expression (Murakami et al., 2000), Hoxa2 could affect chondrogenesis by modulating the expression of Fgfr3. In addition, preliminary real-time PCR data (unpublished results) indicate higher $\operatorname{Sox} 9$ expression rates at birth in Col2a1/Hoxa2-lacZ cartilages as compared with $\beta S$ $H o x a 2-l a c Z$. This might reflect the observed delay in chondrocyte differentiation and cartilage maturation, as Sox9 expression has been shown to decrease during the maturation process ( $\mathrm{Ng}$ et al., 1997). Altogether, these data argue against a direct inhibition of $\operatorname{Sox} 9$ expression by Hoxa2 but rather, as was previously suggested for patterning events (Abzhanov et al., 2003; Bobola et al., 2003), for a negative interference of Hoxa2 on the BMP and FGF pro-chondrogenic signaling cascades (Iimura et al., 1994; Murtaugh et al., 1999; Carlberg et al., 2000; Murakami et al., 2000; Zeng et al., 2002). This interference could thus impair chondrogenesis and delay cartilaginous maturation, eventually leading to a prolonged Sox9 expression.

Bapx1 $(\mathrm{Nkx3.2})$ is an important modulator of the BMP signaling cascade. Bapx $1^{-1-}$ mice (Triboli and Lufkin, 1999) resemble Col2a1/Hoxa2-lacZ mutants: they lack correctly shaped vertebral bodies, bony basihyoid, and supraoccipital due to chondrogenesis failure and they display altered Fgfr3 and Bmp4 expression patterns. In addition, Bapx1 was shown to induce Sox9 expression by mediating the Shh-dependent response of the prechondrogenic mesoderm to BMP signals (Zeng et al., 2002). Moreover, the Xenopus Bapxl homolog is down-regulated in BA1 following a targeted XHoxa2 gain of function (Pasqualetti et al., 2000), suggesting that decreased Bapxl activity may also be involved in the Hoxa2-mediated impairment of chondrogenesis.

Another important finding is that not only endochondral structures but also some membranous bones were affected in Col2a1/Hoxa2-lacZ transgenics. The pterygoid and tympanic ring as well as the whole face were reduced, contrary to what occurs in Col2al KO mice in which only cartilaginous matrix deposition is 
affected ( $\mathrm{Li}$ et al., 1995). This could result from perturbed interactions between growing membranous bones and the adjacent cartilages or from a direct inhibition of membranous ossification following transgene activation into the dermis as suggested by a previous study (Kanzler et al., 1998). The unexpected Col2al-Cre expression in the dermis that presumably results in the crumpled skin of double transgenics newborns is supposed to arise from the ectopic activity of the Col2al promoter fragment contained in the Col2al-Cre transgene. Ectopic activity of integrated transgenes is a commonly observed phenomenon. It is worth noticing that another Col2al-Cre transgenic line has been generated by Sakai et al. (2001) for which nonchondrocytic Cre expression was also reported.

Is the anti-skeletogenic activity specific of Hoxa2 or is it a common property of the entire Hox family? Overexpression of Hoxd4 and Hoxc8 in their own expression domains resulted in a closely similar chondrodysplasia due to an accumulation of proliferating chondrocytes with no further hypertrophic differentiation (Yueh et al., 1998). Hoxc8 was further shown to mediate the BMP-induced osteoblastic differentiation through cooperation with Smad1, an early effector of BMPs, and to participate to the Smad6-dependent negative feed-back loop on BMP signaling (Bai et al., 2000). This has to be paralleled with the observation that mice lacking $P b x l$ exhibit premature differentiation and maturation of cartilages, while no Hox loss of function displayed such a phenotype. This points toward a possible redundancy in the shared ability of Hox proteins to restrain early chondrogenic differentiation.

In conclusion, forcing the expression of Hoxa2 to persist at a stage of chondrogenesis in which it should be normally switched off led to severe chondrodysplasia in transgenic mice. By inducing the conditional expression of Hoxa2 at the onset of chondrocyte differentiation, we demonstrated that, independently of its role in patterning and shaping bones, this gene, presumably like other Hox genes, keeps chondrogenic differentiation under control from its beginning. We therefore propose that Hoxa2 expression is required up to a stage precursor cells assemble in condensations and have to remain undifferentiated, while later on, differentiation and maturation of cartilage occurring, Hoxa2 needs to be silenced.

Acknowledgments We are grateful to F. M. Rijli, X. Lampe, and F. Gofflot for helpful discussions and critical comments on the manuscript. Our thanks are due to Valérie Bonte and Nathalie Pacico for technical assistance. The anti-Hoxa2 raised antibody was kindly provided by A. Nazarali. We are also grateful to P. Soriano and to R. Behringer for providing us with the R26R and Col2alCre recombinant mice, respectively. Finally, we thank J. P. Devogelaer and D. Dienst for their help in pQCT analysis. This work was supported by the National Fund for Scientific Research
(FNRS, Belgium) and the Fonds Spéciaux de recherche (FSR) of the Université catholique de Louvain. L. M. held an FRIA fellowship (FNRS, Belgium) and a FSR grant.

\section{References}

Abzhanov, A., Tzahor, E., Lassar, A.B. and Tabin, C.J. (2003) Dissimilar regulation of cell differentiation in mesencephalic (cranial) and sacral (trunk) neural crest cells in vitro. Development 130:4567-4579.

Akiyama, H., Chaboissier, M.-C., Martin, J.F., Schedl, A. and De Crombrugghe, B. (2002) The transcription factor Sox9 has essential roles in successive steps of the chondrocyte differentiation pathways and is required for expression of Sox 5 and Sox6. Genes Dev 16:2813-2828.

Bai, S., Shi, X., Yang, X. and Cao, X. (2000) Smad6 as a transcriptional corepressor. J Biol Chem 275:8267-8270.

Bi, W., Huang, W., Withworth, D.J., Deng, J.M., Zhang, Z., Behringer, R.R. and De Crombrugghe, B. (2001) Haploinsufficiency of Sox9 results in defective cartilage primordial and premature skeletal mineralization. Proc Natl Acad Sci USA 98:6698-6703.

Bobola, N., Carapuço, M., Ohnemus, S., Kanzler, B., Leibbrandt, A., Neubüser, A., Drouin, J. and Mallo, M. (2003) Mesenchymal patterning by Hoxa 2 requires blocking Fgf-dependent activation of Ptx1. Development 130:3403-3414.

Carlberg, A.L., Pucci, B., Rallapalli, R., Tuan, R.S. and Hall, D.J. (2000) Efficient chondrogenic differentiation of mesenchymal cells in micromass culture by retroviral gene transfer of BMP-2. Differentiation 67:128-138.

Christ, B., Huang, R. and Wilting, J. (2000) The development of the avian vertebral column. Anat Embryol 202:179-194.

Couly, G.F., Coltey, P.M. and Le Douarin, N.M. (1993) The triple origin of skull in higher vertebrates: a study in quail-chick chimeras. Development 117:409-429.

Creuzet, S., Couly, G., Vincent, C. and Le Douarin, N.M. (2002) Negative effect of Hox gene expression on the development of the neural crest-derived facial skeleton. Development 129:4301-4313.

Drobac, E., Durand, E., Laudenbach, V., Mantz, J. and Gallego, J. (2004) A simple method for short-term controlled anesthesia in newborn mice. Physiol Behav 82:279-283.

Gendron-Maguire, M., Mallo, M., Zhang, M. and Gridley, T. (1993) Hoxa2 mutant mice exhibit homeotic transformation of skeletal elements derived from cranial neural crest. Cell 75: $1317-1331$.

Grammatopoulos, G.A., Bell, E., Toole, L., Lumsden, A. and Tucker, A. (2000) Homeotic transformation of branchial arch identity after Hoxa2 overexpression. Development 127: $5355-5365$.

Hoffmann, A., Czichos, S., Kaps, C., Bächner, D., Mayer, H., Zilberman, Y., Turgeman, G., Pelled, G., Gross, G. and Gazit, D. (2002) The T-box transcription factor Brachyury mediates cartilages development in mesenchymal stem cell line C3H10T1/ 2. J Cell Sci 115:769-781.

Huang, Z., Xu, H. and Sandell, L. (2004) Negative regulation of chondrocyte differentiation by transcription factor $A P-2 \alpha$. J Bone Miner Res 19:245-255.

Hunter, M.P. and Prince, V. (2002) Zebrafish Hox paralogue group 2 genes function redundantly as selector genes to pattern the second pharyngeal arch. Dev Biol 247:367-389.

Iimura, T., Oida, S., Takeda, K., Maruoka, Y. and Sasaki, S. (1994) Changes in Homeobox-containing gene expression during ectopic bone formation induced by bone morphogenic protein. Biochem Biophys Res Com 201:980-987.

Kanzler, B., Kuschert, S.J., Liu, Y.H. and Mallo, M. (1998) Hoxa2 restricts the chondrogenic domain and inhibits bone formation during development of the branchial area. Development 125: 2587-2597. 
Kellendonk, C.F., Tronche, F., Monaghan, A.-P., Angrand, P.-O., Stewart, F. and Schütz, G. (1996) Regulation of Cre recombinase activity by the synthetic steroid RU48. Nucleic Acids Res 24:1404-1411.

Krumlauf, R. (1994) Hox genes in vertebrate development. Cell 78:191-201.

Leavitt, J., Gunning, P., Porreca, P., Ng, S.-Y., Lin, C.-S. and Kedes, L. (1984) Molecular cloning and characterization of mutant and wild-type human $\beta$-actin genes. Mol Cell Biol 4:1961-1969.

Lengelé, B., Schowing, J. and Dhem, A. (1996) Embryonic origin and fate of chondroid tissue and secondary cartilages in the avian skull. Anat Rec 246:377-393.

Li, S.W., Prockop, D.J., Helminen, H., Fässler, R., Lapveteläienen, T., Kilary, K., Peltarri, A., Arokoski, J., Lui, H., Arita, M. and Khillan, J. (1995) Transgenic mice with targeted inactivation of the Col2al gene for collagen II develop a skeleton with membranous and periosteal bone but no endochondral bone. Genes Dev 9:2821-2830.

Maconochie, M., Ramachandran, K., Nonchev, S., Maier, P., Manzanares, M., Mitchell, P.J. and Krumlauf, R. (1999) Regulation of Hoxa2 in cranial neural crest cells involves members of the AP-2 family. Development 126:1483-1494.

Murakami, S., Kan, M., McKeehan, W.L. and De Crombrugghe, B. (2000) Up-regulation of the chondrogenic Sox9 gene by fibroblast growth factors is mediated by the mitogen-activated protein kinase pathway. Proc Natl Acad Sci USA 97:1113-1118.

Murtaugh, L.C., Chyung, J.H. and Lassar, A.B. (1999) Sonic Hedgehog promotes somitic chondrogenesis by altering the cellular response to BMP signalling. Genes Dev 13:225-237.

Ng, L.-J., Wheatley, S., Muscat, G.O., Conway-Campbell, J., Bowles, J., Wright, E., Bell, D.M., Tam, P.P., Cheah, K.S. and Koopman, P. (1997) SOX9 binds DNA, activates transcription, and coexpresses with type II collagen during chondrogenesis in the mouse. Dev Biol 183:108-121.

Ovchinnikov, D.A., Deng, J.M., Ogunrinu, G. and Behringer, R.R. (2000) Col2al-directed expression of Cre recombinase in differentiating chondrocytes in transgenic mice. Genesis 26:145-146.

Pasqualetti, M., Ori, M., Nardi, I. and Rijli, F.M. (2000) Ectopic Hoxa2 induction after neural crest migration results in homeosis of jaw elements in Xenopus. Development 127:5367-5378.

Peters, H., Wilm, B., Sakai, N., Imai, K., Maas, R. and Balling, R. (1999) Pax1 and Pax9 synergistically regulate vertebral column development. Development 126:5399-5408.

Rijli, F., Mark, M., Lakkaraju, S., Dierich, A., Dollé, P. and Chambon, P. (1993) A homeotic transformation is generated in the rostral region of the head by disruption of Hoxa2, which acts as a selector gene. Cell 75:1333-1349.
Rodrigo, I., Hill, R.E., Balling, R., Münsterberg, A. and Imai, K. (2003) Paxl and Pax9 activate Bapx1 to induce chondrogenic differentiation in the sclerotome. Development 130:473-482.

Rossi, F., MacLean, H., Yuan, W., Francis, R.O., Semenova, E., Lin, C.S., Kronenberg, H.M. and Cobrinik, D. (2002) p107 and p130 coordinately regulate proliferation, Cbfal expression, and hypertrophic differentiation during endochondral bone development. Dev Biol 247:271-285.

Sakai, K., Hiripi, L., Glumoff, V., Brandau, O., Eerola, R., Vuorio, E., Bösze, S., Fässler, R. and Aszodi, A. (2001) Stage- and tissuespecific expression of a Col2al-Cre fusion gene in transgenic mice. Matrix Biol 19:761-767.

Santagati, F. and Rijli, F.M. (2003) Cranial neural crest and the building of the vertebrate head. Nat Rev Neurosci 4:806-818.

Scheijen, B., Bronk, M., Van der Meer, T. and Bernards, R. (2003) Constitutive E2F1 overexpression delays endochondral bone formation by inhibiting chondrocyte differentiation. Mol Cell Biol 23:3656-3668.

Selleri, L., Depew, M.J., Jacobs, Y., Chanda, S.K., Tsang, K.Y., Cheah, K.S.E., Rubenstein, J.L.R., O'Gorman, S. and Cleary, M.L. (2001) Requirement for Pbxl in skeletal patterning and programming chondrocyte proliferation and differentiation. Development 128:3543-3557.

Shaw-Jackson, C. and Michiels, T. (1999) Absence of internal ribosome entry site-mediated tissue specificity in the translation of a bicistronic transgene. J Virol 73:2729-2738.

Soriano, P. (1999) Generalized lacz expression with the ROSA26 Cre reporter strain. Nat Genet 21:70-71.

Stadler, H.S., Higgins, K.M. and Capecchi, M.R. (2001) Loss of Eph-receptorexpression correlates with loss of cell adhesion and chondrogenic capacity in Hoxa13 mutant limbs. Development 128:4177-4188

Triboli, C. and Lufkin, T. (1999) The murine Bapx1 homeobox gene plays a critical role in embryonic development of the axial skeleton and spleen. Development 126:5699-5711.

Wan, M., Shi, X., Feng, X. and Cao, X. (2001) Transcriptional mechanisms of bone morphogenetic protein-induced osteoprotegrin gene expression. J Biol Chem 276:10119-10125.

Yang, Y., Topol, L., Lee, H. and Wu, J. (2003) Wnt5a and Wnt5b exhibit distinct activities in coordinating chondrocyte proliferation and differentiation. Development 130:1003-1015.

Yueh, Y.G., Gardner, D.P. and Kappen, C. (1998) Evidence for regulation of cartilage differentiation by the homeobox gene Hoxc-8. Proc Natl Acad Sci USA 95:9956-9961.

Zeng, L., Kempf, H., Murtaugh, L.C., Sato, M.E. and Lassar, A.B. (2002) Shh establishes an Nkx3 2/Sox9 autoregulatory loop that is maintained by BMP signals to induce somatic chondrogenesis. Genes Dev 16:1990-2005. 